\title{
Novel Pre Treatment Techniques for Extraction of Fermentable Sugars from Natural Waste Materials for Bio Ethanol Production
}

\author{
Megha Srivastava, Shubhalakshmi Sengupta, Papita Das* and Siddhartha Datta \\ Department of Chemical Engineering, Jadavpur University, India
}

Submission: December 06, 2017; Published: December 14, 2017

*Corresponding author: Papita Das, Department of Chemical Engineering, Jadavpur University, 188, Raja S. C. Mullick Road, Kolkata-700032, India, Email: papitasaha@gmail.com

\begin{abstract}
The demand of traditional/domestic fuel is increasing day by day. Bio ethanol, a non-conventional source of energy is a solution to this problem. India stands second in sugarcane production worldwide, so a huge amount of agriculture waste residue is produced. This study presents the extraction and analytical estimation of cellulose and hemi cellulose of sugarcane bagasse and extraction of soluble sugars from it for bio ethanol production. At first samples were prepared and analysed for bulk density moisture content, ash content, volatile matter content, fixed carbon content and calorific value. Cellulose and hemi cellulose estimated after the treatments suggested their efficient extraction from the sugarcane bagasse. Different pre treatment technique are performed to increase the amount of fermentable sugars and to decrease the lignin content present in bagasse. Then the pre-treated bagasse is placed for enzyme hydrolysis followed by fermentation to produce bio ethanol. The result suggested that waste bagasse can be used as a renewable source of energy for bio ethanol/bio fuel production in an environmentally sustainable and economically viable way.
\end{abstract}

Keywords: Sugarcane Bagasse Cellulose; Hemi Cellulose; Lignin; Bio Ethanol

Abbreviations: SEM: Scanning Electron Microscopy; TEM: Transmission Electron Microscopy; FTIR: Fourier Transform Infrared; NDF: Neutral Detergent Fibre; ADF: Acid Detergent Fibres

\section{Introduction}

Rapid industrialization increases the demand of fossil fuels. This causes fuel crises and it affects our environment. Air pollution is responsible for several major problems like global warming, acid rain, and the deterioration of the ozone layer. The list of the pollutants includes gases like carbon dioxide, carbon monoxide, nitrogen dioxide, sulphur dioxide etc. Emission of carbon monoxide is higher due to incomplete combustion of fuels. The increasing concentration of gas makes it hard for our body parts to get oxygen they need to run correctly. Air pollution can result from the burning of fossil fuels, such as coal, oil, natural gas, and gasoline to produce electricity and power our vehicles. In the similar way nitrogen dioxide which mostly comes from power plants and cars react in the atmosphere to form acid rain. Bio ethanol, a non-conventional source of energy is a solution to this problem. Ethanol when used as a fuel as it offers many advantages such as it has low price, and comparatively less emissions than gasoline.

Ethanol contains 35\% oxygen that helps complete combustion of fuel and reduces particulate emissions that poses health hazard to living beings. Ethanol has a high octane number (99) than petrol (80-100). When ethanol is added in small quantities to unleaded petrol, it acts as an octane booster replacing the conventional additives for this purpose (Meta tertiary butyl ether, which can create adverse health effects). For this reasons ethanol is used widely as a fuel [1]. There are three types of bio fuels: 1st, 2nd and 3rd generation bio fuels based on their source of biomass and limitations as renewable source of energy. First Generation bio ethanols are produced directly from food crops including corn, sugarcane, barley etc. by fermentation. Second Generation bio ethanols are produced from non-food crops such as agriculture residue, wood, organic waste, food crop waste and specific biomass crops. The Third Generation of bio fuels is produced from microbial biomasses like algal bio mass [2]. Second generation ethanol production from lingo cellulosic materials Ligno cellulosic material shows a promising option in ethanol production due to their output/input energy ratio, availability, low cost and higher ethanol yields. Renewable 'plant biomass' refers particularly to cheap and abundant non-food lingo cellulose-rich materials which comes from the plants.

In countries like India, a huge amount of waste generated from agricultural production of various crops like cotton, mustard, chilli, sugarcane, sorghum, sweet sorghum, pulses, oilseeds, etc. that do not find any alternative use and are either 
left in the fields or are burned. Hence, these could be used in bio ethanol production which is a good alternative to use it in an environmentally friendly manner. Use of agricultural residues helps in reduction of deforestation as our reliance on forest woody biomass decreases. Short harvest period of crop residues preferred them more consistently available to bio ethanol production $[3,4]$. Production of bio ethanol from lingo cellulosic biomass is still a challenge because of its very complex structure where cellulose and hemicelluloses are formed a complex matrix with lignin. In grain ethanol processes, the fermentable monomeric sugars are liberated from the grain starch and in cellulosic processes, the fermentable sugars are the cellulose and hemicelluloses [5]. Ligno cellulosic materials consist mainly of three polymers which are cellulose, hemi cellulose, and lignin.

These polymers are associated with each other in a heteromatrix to different degrees and varying relative composition depending on species, type and source of the biomass. The main objectives of the pre-treatment process are to speed up the rates of hydrolysis and increase the yields of fermentable sugars. In all pre- treatment processes, these goals are accomplished by modifying the structure of the polymer matrix in the biomass, thus making the carbohydrate fractions more susceptible to acid attack or more accessible to enzyme action reported that the main processing challenge in the ethanol production from lingo cellulosic biomass is the feedstock pre-treatment. Pre treatment is done to reduce the crystallinity of cellulose and increase the fraction of amorphous cellulose, and to break the matrix of cellulose and lignin bound by hemi cellulose should be broken to reduce the, the most suitable form for enzymatic attack [6].

Suggested that though the combination of grinding with other pre treatment method reduces the crystallinity of the biomass superfine grinding of biomass with steam treatment showed better than ground residue when hydrolyzed $[7,8]$. Chemical pre treatment involving dilute acid and alkali are also sought after pre treatment technologies Sugar cane bagasse is a waste of the sugar industry and a cheap source of lingo cellulosic material for extraction of fermentable sugars for bio ethanol production. Suggested that sodium hydroxide $(\mathrm{NaOH})$ presents the greatest degradation and subsequent fermentation yields with compared to other alkalis, such as sodium carbonate, ammonium hydroxide, calcium hydroxide and hydrogen peroxide [9] used $\mathrm{NaOH}$ solution to treat the pith component of sugarcane bagasse $(0.2 \mathrm{~g}$ of $\mathrm{NaOH}$ per pith gram) and obtaining a maximum digestibility of $71 \%$ at $92^{\circ} \mathrm{C}$. Described that acids hydrolyze hemicelluloses thus produce a liquid phase rich in xylose, with minor amounts of lignin derivatives so it is an outstanding method for hemi cellulose recovery and it has been successfully applied to sugarcane bagasse [10].

Again, found that different pre treatment methods have singular action mechanisms. They either decrease cellulose crystallinity or the degree of polymerization. They increase accessible surface areas or selectively remove hemi cellulose and lignin from the lingo cellulosic matrix. So an effective pre treatment strategy is needed to minimize carbohydrate degradation and the production of enzyme inhibitors and toxic products for fermenting microorganisms [11]. Thus in this study novel techniques have been applied to extract fermentable sugars from sugarcane bagasse. Different acid and alkali pre treatments were done to remove the lignin and hemi cellulose fractions and the most effective technique was obtained based on the characterization analysis of fourier transform infrared (FTIR) spectroscopy, scanning electron microscopy (SEM) and transmission electron microscopy (TEM) and estimation of the cellulose, lignin and hemi cellulose fractions of the pre treated samples. Fermentable sugars were then extracted from the pre treated samples by enzymatic degradation by micro organisms. A marine fungus (Aspergillus $\mathrm{sp}$ ) was used for the first time in this study. The sugars were then fermented further to obtain bio ethanol.

\section{Materials and Methods}

\section{Materials}

Sugarcane bagasse was obtained from Midnapore, West Bengal. Sodium sulphite (Merck, India), Decarbohydro naphthalene (Merck, India), Tween 80 (Himedia, India), Disodium ethylene-diamine-tetraacetate (Himedia, India), Sodium borate decarbohydrate (Himedia, India), Sodium lauryl sulphate (Merck, India), ethoxy ethanol (Merck, India), Acetone(Merck, India), Cetyltrimethyl ammonium bromide (Himedia, India), Acetic acid (Merck, India), Nitric acid (Merck, India), Dextrose (Merck, India), Cellulose powder(Merck, India), Sodium hydroxide (Merck, India), Hydrochloric acid (Merck, India), Iodine solution (Merck, India), Potassium iodide (Merck, India), Potassium dichromate (Merck, India), Sodium chlorite (Merck, India), Decaline (Merck, India) , Carboxy Methyl Cellulose, CMC (Merck, India), Peptone (Merck, India), Sodium nitrate (Merck, India), Potassium Chlorite (Merck, India), Magnesium Sulphate (Merck, India), Ferrous Sulphate (Merck, India), Dipotassium hydrogen phosphate (Merck, India), Ammonium Sulphate (Merck, India), Agar, yeast extract (Merck, India), Dinitrosalicylic acid, DNS (Merck, India). Aspergillus sp. Strain was isolated from marine waters of west bengal and Saccharomyces cerevisiae strain (MTCC 170) was procured from MTCC, pune.

Preparation of Raw Material: The raw bagasse was received at about 30\% moisture content. It was sun-dried for 4-5 days and finely ground by hammer milled .The sugarcane bagasse was chopped in small pieces and was placed in sterilized petriplates and dried in a hot air oven at 80 degree celcius temperatures till constant weight. It was immediately grounded in a mixer and stored in polypropylene bags for subsequent uses.

Physicochemical Analysis of Raw Sugarcane Bagasse: Samples of sugarcane bagasse dry were taken for its characterization which was to analyse for moisture, density, ash content, volatile matter, fixed carbon and calorific value in accordance with ASTM D 1037 (1991), ASTM D 2017 (1998) and ISO 562/1974. 


\section{Pre treatment Techniques}

Milling treatment: Chipped and grinded bagasse was put into $250 \mathrm{ml}$ Erlenmeyer flask. It was then moistened with distilled water. The flasks were incubated for $2 \mathrm{~h}$ at room temperature.

Alkaline Treatment: The bagasse sample was pre treated with different alkaline concentration ranging from $0.5 \%$ to $5 \%$ $\mathrm{NaOH}$ solution. The alkaline pre treatment was done in two different methods. In first method the bagasse was pre treated with $\mathrm{NaOH}$ solutions of different concentrations at $121 \mathrm{oC}$ and 15 psi pressure for 1 hour at the ratio of 1:10 (1 gram of substrate with $10 \mathrm{ml}$ of $\mathrm{NaOH}$ solution). The pre treated bagasse was washed with tap water until the $\mathrm{pH}$ of the filter reached 7 . The washed bagasse was dried at $6000 \mathrm{C}$ overnight to constant weight and stored at room temperature in air tight container for further use. In second method, the bagasse was treated in the same process except auto clave; it was kept in room temperature for 15 minutes.

Steam Treatment of Milled Alkaline Sugarcane Bagasse: Ten gram of chipped and grinded bagasse were put into $250 \mathrm{ml}$ Erlenmeyer flask then moistened with distilled water. The flasks were steam treated by autoclaving at $121 \mathrm{oC}$ and 1.5 bars for 20 min. then extraction, filtration and determination of (TRS) were performed as previously mentioned.

Acid Treatment: The bagasse sample was pre treated with different acid concentration ranging from $0.5 \%$ to $4 \%$ H2SO4 solution. In this method the bagasse was pre treated with $\mathrm{H} 2 \mathrm{SO} 4$ solutions of different concentrations at room temperature for 1 hour at the ratio of 1:10 ( 1 gram of substrate with $10 \mathrm{ml}$ of $\mathrm{H} 2 \mathrm{SO} 4$ solution) as per method of the pre treated bagasse was washed with tap water until the $\mathrm{pH}$ of the filter reached 7 . The washed bagasse was dried at $600 \mathrm{C}$ overnight to constant weight and stored at room temperature in air tight container for further use [12].

Alkali and Acid Treatment: Different alkali pre treated bagasse samples were again treated with $1 \% \mathrm{H}_{2} \mathrm{SO}_{4}$ solutions of different concentrations at room temperature for 1 hour at the ratio of 1:10 (1 gram of substrate with $10 \mathrm{ml}$ of $\mathrm{H}_{2} \mathrm{SO}_{4}$ solution) as per method of. The pre treated bagasse was washed with tap water until the $\mathrm{pH}$ of the filter reached 7. The washed bagasse was dried at 600C overnight to constant weight and stored at room temperature in air tight container for further use.

\section{Characterizations of The Pre treated Samples}

Fourier Transform Infrared (FTIR) Spectroscopy: The infrared spectra (wave numbers in $\mathrm{cm}-1$ ) were obtained on a Magma - IR 560 E.S.P -Perkin Elmer spectrophotometer, by means of a $\mathrm{KBr}$ disk containing $3 \%$ finely ground samples.

Scanning Electron Microscopy (SEM): Scanning electron microscopy (SEM - FEI / Inspect S50 model) was used to observe modifications on bagasse fibres. Samples were adhered to carbon tape and sputter coated with gold (sputter Emitech / K550 model) and observed in the SEM through the use of an acceleration voltage of $20 \mathrm{KV}$ and working distance of around $38 \mathrm{~mm}$. Hundreds of SEM images were obtained on different areas of the samples to guarantee the reproducibility of the results.

Transmission Electron Microscopy (TEM): TEM (JEM 2100 HR, JEOL, Japan) with energy-dispersive X-ray spectroscopy (EDS) was used with a field emission gun; this provided high resolution operation at $200 \mathrm{kV}$ and $1.05 \mathrm{~A}$.

\section{Estimation of Pre treated Sugarcane Bagasse}

Cellulose, Hemi cellulose and Lignin content determination: In a refluxing flask, powdered sample was mixed with cold neutral detergent solution. The neutal detergent solution is prepared as follows. Disodium-ethylene di amine-tetra acetate and sodium borate de carbohydrate were dissolved in distilled water by heating and to this added sodium lauryl sulphate and ethoxy ethanol. A solution 4.5\% Na2HPO4 was then added to the mixture. De carbo hydro napthalene and sodium sulphite was then added to mixture of sugarcane bagasse sample and cold neutral detergent solution, which was then heated to boiling and refluxed for 1 hour. The contents were filtered through sintered glass crucible (G-2) and washed with hot water. The contents were finally washed with acetone twice and the residue transferred to a crucible. The sample was dried at $100 \mathrm{oC}$ for 8 hour, cooled in a decicator and weighed. The residue was designated as neutral detergent fibre (NDF). To calculate hemi cellulose content, the amount of acid detergent fibres (ADF) was subtracted from the amount of NDF.

Cellulose Estimation By Anthrone Method: Cellulose of the sugarcane bagasse samples undergoes acetolysis with acetic acid/nitric reagent forming acetylated cello dextrine which gets dissolved and hydrolysed to form glucose molecules on treatment with $67 \% \mathrm{H} 2 \mathrm{SO}$. This glucose molecule is dehydrated to form hydroxyl methyl furfural which forms green coloured product with anthrone and the colour intensity is measured at $630 \mathrm{~nm}$ in spectrophotometer (Perkin Elmer, Germany).

\section{Extraction Of Fermentable Sugars Using Aspergillus sp.}

Inoculum preparation: The isolated and identified fungi culture was sub cultured on Czapek modified medium (CMM) with agar containing $2 \% \mathrm{CMC}, 0.2 \%$ peptone, $0.2 \% \mathrm{NaNO}_{3}, 0.05 \%$ $\mathrm{KCl}, 0.05 \% \mathrm{MgSO}_{4}, 0.001 \% \mathrm{FeSO}_{4}, 0.1 \% \mathrm{~K}_{2} \mathrm{HPO}_{4}$ and $1.7 \%$ agar and incubated at $30 \mathrm{oC}$. Fully sporulated plates were obtained after 6 days. The sporulated plated were flooded with $20 \mathrm{ml}$ of distilled water containing $0.1 \%$ Tween 80 . Spores were dislodged by gentle pipetting. The resulting spore suspension was used as inoculum.

Extraction of fermentable sugars: Five grams of sugarcane bagasse (pre treated samples) was weight into $250 \mathrm{ml}$ Erlenmeyer flasks and moistened with basal medium containing $0.2 \% \mathrm{NaNO}_{3}$, $0.05 \% \mathrm{KCl}, 0.05 \% \mathrm{MgSO}_{4}, 0.001 \% \mathrm{FeSO}_{4}, 0.1 \% \mathrm{~K}_{2} \mathrm{HPO}_{4}$. Peptone was added to the above media as nitrogen source. The flasks were inoculated with $5 \mathrm{ml}$ spore suspension per gram dry weight of substrate. The inoculated substrate was mixed thoroughly and incubated statically at 300C. 
Estimation of fermentable sugars: The solid material was then mixed vigorously with $100 \mathrm{ml}$ distilled water for extraction of soluble reducing sugar, then filtered with cloth sheets to separate the content into solid and liquid parts. The liquid filtrate was centrifuge at $10,000 \mathrm{rpm}$ for $10 \mathrm{~min}$, and then, the content of total reducing sugars (TRS) was determined in clear supernatant by DNS (dinitro salicylic acid) method.

\section{Bio ethanol Production}

The production medium was formulated according to where fermentation media was added to the hydrolysate obtained from fungal isolate through solid state fermentation process and then sterilized by autoclaving at $121 \mathrm{oC}$ for $20 \mathrm{~min}$ [13]. the medium was inoculated with pre-selected yeast isolates. The inoculated cultures were incubated at $30 \mathrm{oC}$ for $48 \mathrm{~h}$ at $150 \mathrm{rpm}$. The fermentation media was prepared by adding $0.1 \% \mathrm{MgSO}_{4}, 0.2 \%$ $\mathrm{KH}_{2} \mathrm{PO}_{4}, 0.3 \%\left(\mathrm{NH}_{4}\right)_{2} \mathrm{SO}_{4}, 0.3 \%$ peptone and $0.4 \%$ yeast extract to the enzymatic hydrolysate and filter sterilized. Initial $\mathrm{pH}$ was adjusted to 5. A $12 \mathrm{~h}$ old seed culture of Saccharomyces cerevisiae (MTCC 170) was inoculated into the fermentation medium at $5 \%(\mathrm{v} / \mathrm{v})$ ratio. Fermentation was carried out at $30 \mathrm{o} \mathrm{C}$ in static condition. Samples were collected at regular intervals and centrifuge for $10 \mathrm{~min}$ at $4 \mathrm{oC}$ and $12000 \mathrm{rpm}$ and supernatant were taken for estimation of ethanol. Ethanol was extracted from the fermentation medium by a rotator evaporator (Buchi, India). The ethanol-water extract was used for further estimation of ethanol content according to a method by [14].

\section{Estimation of Ethanol Content}

Spectro photometric method: Alcoholic sample was added directly to the distillation flask, diluted then distilled. Distillation was carried out at 70+20C and the distillate was collected in volumetric flask containing potassium dichromate solution. The contents in the volumetric flask were heated at $600 \mathrm{C}$ in a water bath for 20 minutes. After mixing and cooling the contents of the flask, the absorbance was recorded at $600 \mathrm{~nm}$. The amount of ethanol in each sample was determined by using the standard curve of ethanol.

\section{Results and Discussions}

Table 1: Physical properties of sugarcane bagasse.

\begin{tabular}{|c|c|}
\hline Proximate analysis & Results \\
\hline Bulk Density & $86.6 \mathrm{~kg} / \mathrm{m} 3$ \\
\hline Moisture & $11 \%$ \\
\hline ASH & $3.7 \%$ \\
\hline Volatile Matter & $14 \%$ \\
\hline Fixed Carbon & $82.3 \%$ \\
\hline
\end{tabular}

Characterization of Sugarcane Bagasse: The characterization of the raw bagasse was carried out to determine its physical and chemical properties. The physical properties are given in (Table 1).

\section{Estimation of Raw Bagasse and Pre treated Samples}

Cellulose estimation of Pre-treated sample: The percentages of alkali and acid treatment and acid alkali treatment which showed the best optimized results by spectroscopic analysis using anthrone estimation were presented in (Figure 1). The better extraction of cellulose was observed using both the treatment of acid followed by alkali treatment.

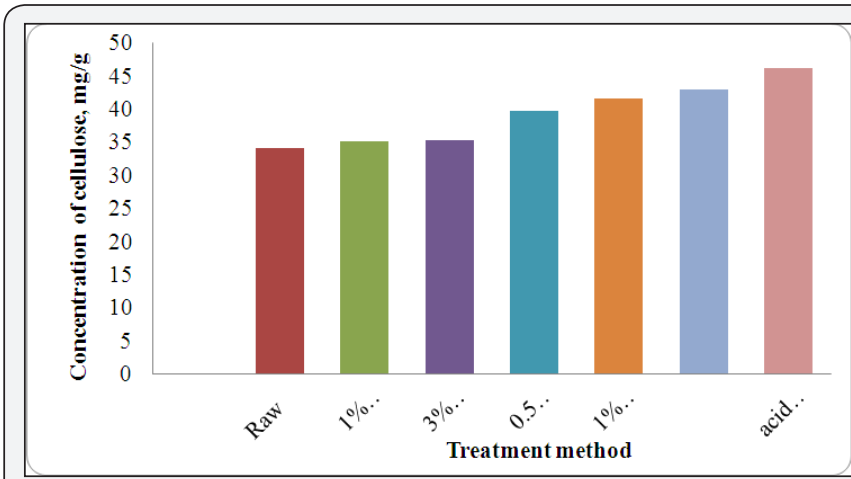

Figure 1: Concentration of cellulose after different pretreatment.

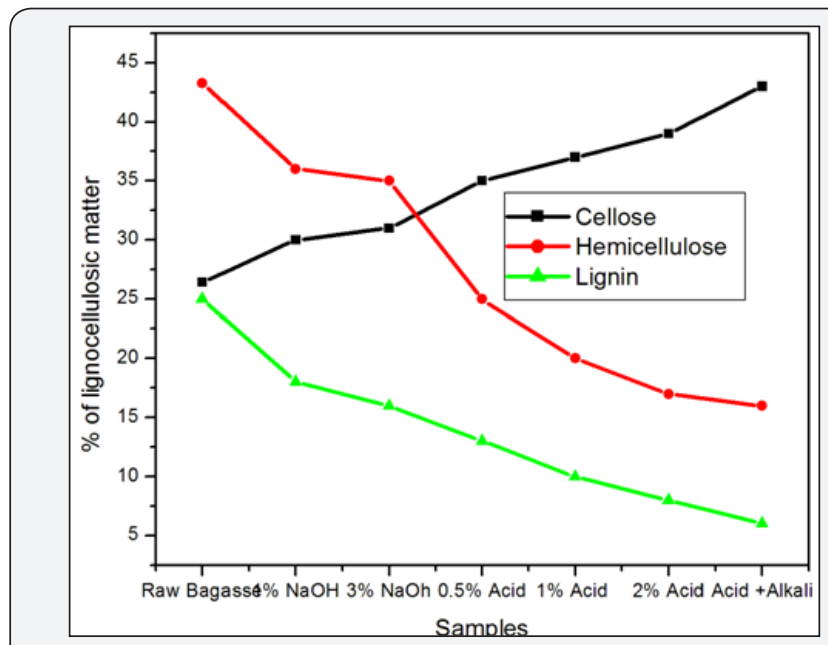

Figure 2: Extraction of cellulose, hemicellulose and lignin using different pre-treatment method.

Estimation of Cellulose, Hemi cellulose and lignin Estimation of Raw Bagasse and Pretreaed Samples by ADF and NDF Method: The ADF and NDF method was applied on the pre treated and after treatment sugarcane bagasse to estimate the cellulose, hemi cellulose and lignin content (Figure 2). The results revealed that efficient hemi cellulose and lignin removal along with increased in cellulose content was observed in case where both acid and alkali treatment was done. Cellulose extracted about $35-43 \%$ through pre treatment method and in case of acidalkali treatment, $43 \%$ cellulose can be extracted from sugarcane bagasse. Thus from the cellulose estimation study, it could be inferred acid and alkali treated samples showed effective lignin fraction removal and increased in cellulose content which can be the best source for extraction of fermentable sugars from waste materials. 


\section{Fourier Transform Infra Red (FTIR) spectroscopy}

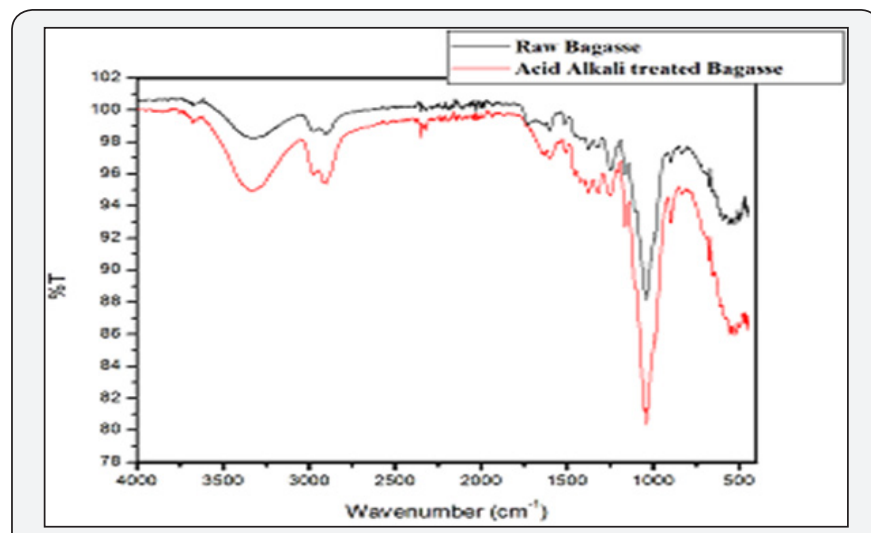

Figure 3: FTIR of raw bagasse and acid alkali pretreated bagasse.

The FTIR analysis of the raw bagasse and acid alkali treated bagasse is given in (Figure 3). The best treatment method observed from the previous analyses was chosen for FTIR analysis. The FTIR figure revealed characteristic feature of a lingo cellulosic material [15]. On treatment with acid alkali, a characteristic peak of $(\beta)$ glycosidic bond corresponding to that of cellulose at about 900 $\mathrm{cm}^{-1}$ was found. This bond is attributed to b-glycosidic linkages between the sugar units. When the raw bagasse is examined, the band of $895 \mathrm{~cm}^{-1}$ is not notable, mainly due to the coverage of cellulose by hemi cellulose and lignin matrix. Thus the treatment resulted in disruption of hemicelluloses and lignin and revealed this characteristic peak of cellulose.

\section{Scanning Electron Microscopy (SEM) Analysis}

Scanning electron microscope (SEM) images of the raw bagasse and the pre treated bagasse are demonstrated in (Figure 4). In the SEM micrograph of raw bagasse, a complete and compact lingo cellulosic structure is clearly observed in (Figure 4A). After undergoing pre treatment by acid and alkali (The treatment which gave best result), the structure of bagasse has been damaged to a certain extent. It is mainly observed in the case of acid with alkali treatment where major cracks are seen on the bagasse surface (Figure 4B). The disruption of the lingo cellulosic structure becomes more pronounced and some tiny holes are exhibited on the surface of pre treated sample. So with this pre treatment method, the lingo cellulosic structure of bagasse has been destroyed in a significant way and smaller cellulosic structures were revealed.

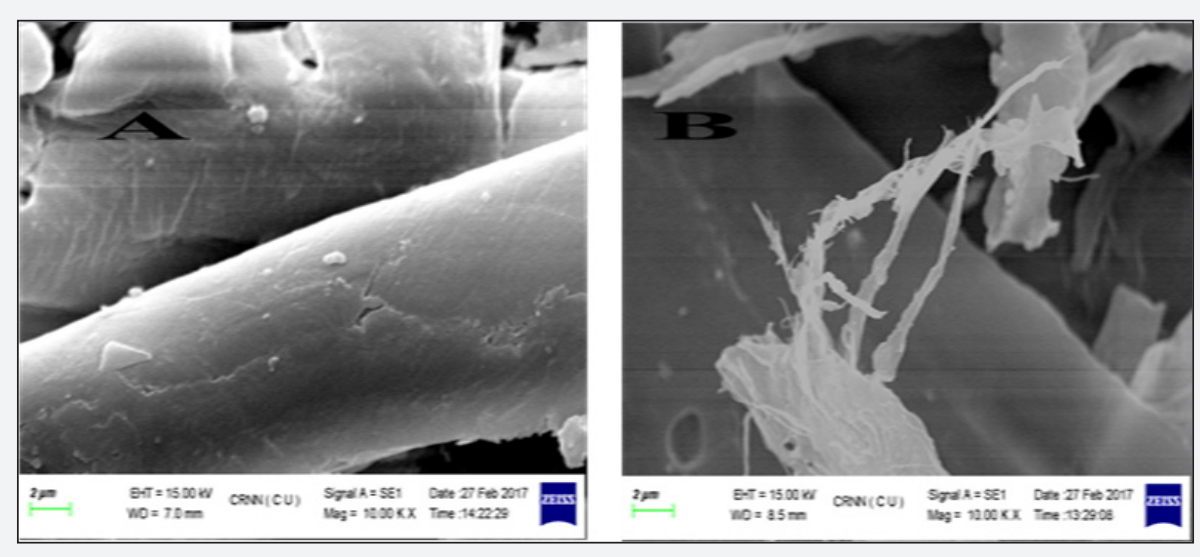

Figure 4: SEM image of $(A)$ raw bagasse and $(B)$ acid alkali pretreated bagasse.

\section{Transmission Electron Microscopy (TEM) Analysis}

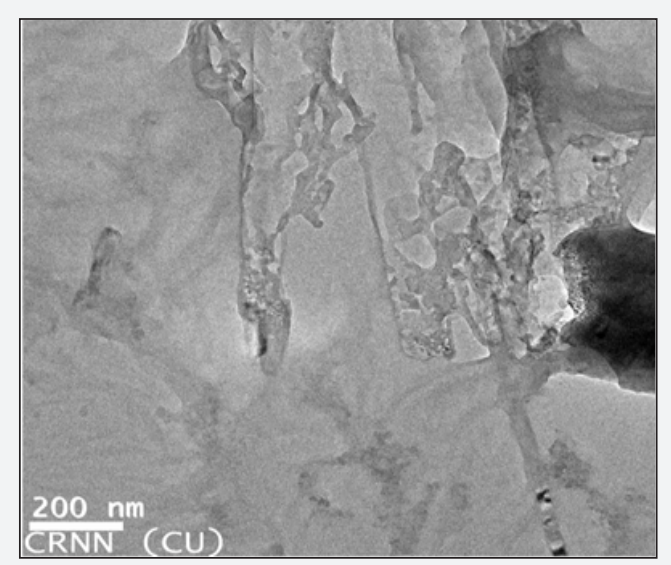

Figure 5: TEM of acid alkali pre treated sugarcane bagasse.
In Figure 5, the TEM micrograph of acid alkali treated sugarcane bagasse revealed reduction of size in the treated samples and also showed characteristic cellulose fibre like structures. Thus, from all the above analysis and observations it could be inferred that effective pre-treatment is required for extraction of fermentable sugars from sugarcane bagasse. Milling of raw bagasse followed by acid plus alkali treatment gave the best results. This treatment reduced the lignin and hemi cellulose content considerably and increased the extraction of the cellulosic fractions along with reduction in cellulose size. This pre treated sugarcane bagasse samples with steam treated alkali, acid and acid plus alkali were further evaluated for production of fermentable sugars.

\section{Enzyme Hydrolysis}

The efficiency of pre treated samples to produce fermentable sugars was evaluated by measuring the total amount of glucose 
(TRS) released from the samples after 48 hours of enzymatic hydrolysis using Aspergullus sp. strain. The applied pre treatment showed different effects on the total reducing sugar yield for the bagasse. Data of different pre treated samples were calculated from standard curve of glucose concentration $(\mathrm{mg} / \mathrm{ml})$ showed the maximum yield of TRS in the case of acid plus alkali treated bagasse sample (figure not shown). The hydrolysis yield (or percentage of cellulose conversion) was calculated for the total process (total hydrolysis yield).Considering the concentration of glucose, total hydrolysis yields reach maximum values between 8 to $9 \mathrm{mg} / \mathrm{ml}$ for acid alkali treated samples ,due to the large increase in cellulose accessibility in this sample. In the case of $2 \%$ acid treatment the glucose yield reached up to $7-7.5 \mathrm{mg} / \mathrm{ml}$ which also can be used for fermentation process for bio ethanol production.

\section{Bio-Ethanol Production}

Bio-ethanol production was carried out with the fermentable sugars extracted from pre-treated sugarcane bagasse. The extracted fermentable sugars was fermented in anaerobic condition using S. Cerevisae stain (MTCC 170) (Figure 6) represented the concentration of ethanol obtained from different pre-treated bagasse reducing sugars. Different pre treatment procedure such as milling, alkali and acid treatment was performed to produce ethanol from reducing sugars extracted from sugarcane bagasse best results was obtained from a combination of pre treatment method where milling, acid and alkali treatment had been applied on the raw sugarcane bagasse. The maximum bio ethanol production using the technology was obtained as $25.33 \%$ after fermentation of the reducing sugars whereas from dextrose, the bio ethanol production was $33 \%$.

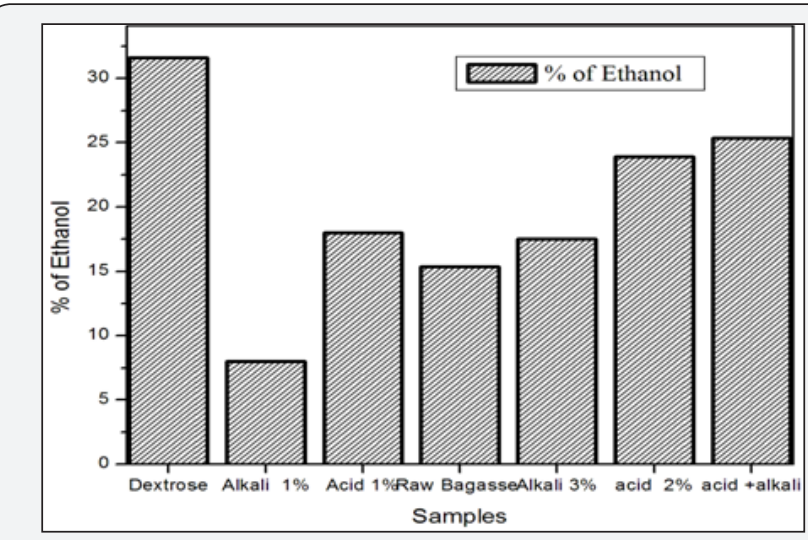

Figure 6: Ethanol production from different pretreated samples.

\section{Conclusion}

This experiment leads us to the conclusion that bio ethanol can be produced from waste materials like sugarcane bagasse and using the pre treatment method, hemi cellulose and lignin fraction can be removed from the raw materials. It can also be concluded that Raw bagasse with milling and acid alkali pre treatment leads to better extraction of cellulose from which higher amount of reducing sugars could be extracted. The reducing sugars extracted from these pre treated samples results production of ethanol. The highest ethanol percentage in ethanol water mix was obtained about $26 \%$ in this study.

\section{Acknowledgement}

SS would like to thank UGC D.S. Kothari Postdoctoral Fellowship scheme (Sanction. No. CH/15-16/0163) for providing financial assistance. Authors are thankful to Prof Aniruddha Mukhopadhyay, Department of Environmental Science, University of Calcutta, Kolkata for the characterization facilities.

\section{References}

1. Balata M, Balata H, Oz C (2008) Progress in bioethanol processing. Progress in Energy and Combustion Science 34(5): 551-573.

2. Caputi A, Ueda MT, Brown T (1968) Spectro photometric determination of ethanol in wine. American Journal of Enol Vitic 19(3): 160-165.

3. Carere CR, Sparling R, Cicek N, Levin DB (2008) Third generation bio fuels via direct cellulose fermentation. Int J MolSci 9(7): 1342-1360.

4. Chandra RP, Bura R, Mabee WE, Berlin A, Pan X, et al. (2007) Substrate pre treatment: the key to effective enzymatic hydrolysis of ligno cellulosics. Adv Biochem Eng Biotechnol 108: 67-93.

5. Himmel ME, Ding SY, Johnson DK, Adney WS, Nimlos MR, et al. (2007) Biomass recalcitrance: engineering plants and enzymes for biofuels production. Science 315(5813): 804-807.

6. Jin S, Chen H (2006) Superfine grinding of steam-exploded rice straw and its enzymatic hydrolysis. Biochem Eng J 30(3): 225-230.

7. Kim S, Dale BE (2004) Global potential bio ethanol production from wasted crops and crop residues. Biomass Bio energ 26(4): 361-375.

8. Knauf M, Moniruzzaman M (2004) Ligno cellulosic biomass processing: a perspective. Int Sugar J 106(1263): 147-150.

9. Kumar P, Barrett DM, Delwiche MJ, Stroeve P (2009) Methods for pre treatment of ligno cellulosic biomass for efficient hydrolysis and bio fuel production. Ind Eng Chem Res 48(8): 3713-3729.

10. Lavarack BP, Griffin GJ, Rodman D (2002) The acid hydrolysis of sugarcane bagasse hemi cellulose to produce xylose, arabinose, glucose and other products. Biomass Bioenergy 23(5): 367-380.

11. Limayema A, Ricke SC (2012) Ligno cellulosic biomass for bio ethanol production: current perspectives, potential issues and future prospects. Prog Energy Combust Sci 38(4): 449-467.

12. Lynd LR, Weimer PJ, van Zyl WH, Pretorius IS (2002) Microbial cellulose utilization: fundamentals and biotechnology. Microbiol Mol Biol Rev 66(3): 506-577.

13. Rodríguez-Vázquez R, Villanueva-Ventura G, Rios-Leal E (1992) Sugarcane bagasse pith dry pretreatment for single cell protein production. Bio resour Technol 39(1): 17-22.

14. Wati L, S Kumari, BS Kundu (2007) Paddy straw as substrate for ethanol production. Ind J Microbiol 46(1): 26-29.

15.Yu J, Zhang X, Tan T (2009) Optimization of media conditions for the production of ethanol from sweet sorghum juice by immobilized Saccharomyces cerevisiae . Biomass and Bio energy 33(3): 521-526. 


CC
Commons Attribution 4.0 Licens
DOI: 10.19080/IJESNR.2017.07.555713

\begin{tabular}{|l|}
\hline \multicolumn{1}{|c|}{ Your next submission with Juniper Publishers } \\
will reach you the below assets \\
- Quality Editorial service \\
- Swift Peer Review \\
- Reprints availability \\
- E-prints Service \\
- Manuscript Podcast for convenient understanding \\
- Global attainment for your research \\
- Manuscript accessibility in different formats \\
( Pdf, E-pub, Full Text, Audio) \\
- Unceasing customer service \\
Track the below URL for one-step submission \\
https://juniperpublishers.com/online-submission.php
\end{tabular}

